\title{
Over-expression of cyclooxygenase-2 in endoscopic biopsies of ectopic gastric mucosa
}

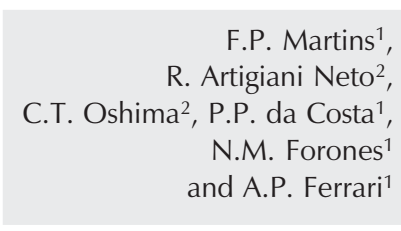

\author{
'Disciplina de Gastroenterologia, 2 Departamento de Patologia, \\ Escola Paulista de Medicina, Universidade Federal de São Paulo, \\ São Paulo, SP, Brasil
}

\begin{abstract}
Correspondence
F.P. Martins

Alameda Casa Branca, 1025, Apto. 21

01408-001 São Paulo, SP

Brasil

E-mail: fernandaprata@terra.com.br

Publication supported by FAPESP.
\end{abstract}

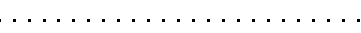

Received November 27, 2006 Accepted July 18, 2007

\begin{abstract}
Ectopic gastric mucosa (EGM) is considered to be a congenital condition. Rare cases of adenocarcinoma have been described. There are no data justifying regular biopsies or follow-up. Cyclooxygenase$2(\mathrm{COX}-2)$ is a protein involved in gastrointestinal tumor development by inhibiting apoptosis and regulating angiogenesis. The aim of this prospective study was to evaluate COX-2 expression in EGM and compare it with normal tissue and Barrett's esophagus. We evaluated 1327 patients. Biopsies were taken from the inlet patch for histological evaluation and from the gastric antrum to assess Helicobacter pylori infection. Biopsies taken from normal esophageal, gastric antrum and body mucosa and Barrett's esophagus were retrieved from a tissue bank. EGM biopsies were evaluated with respect to type of epithelium, presence of $\mathrm{H}$. pylori, and inflammation. COX-2 was detected by immunohistochemistry using the avidin-biotin complex. EGM islets were found in 14 patients $(1.1 \%)$. Histological examination revealed fundic type epithelium in $58.3 \%$ of cases, $\mathrm{H}$. pylori was present in 50\% and chronic inflammation in 66.7\%. Expression of COX-2 was negative in normal distal esophagus, normal gastric antrum and normal gastric body specimens (10 each). In contrast, EGM presented over-expression of COX-2 in $41.7 \%$ of cases and Barrett's esophagus in $90 \%$ of cases ( $\mathrm{P}=0.04$ and 0.03 , respectively). COX-2 immunoexpression in EGM was not related to gender, age, epithelium type, presence of inflammation or intestinal metaplasia, $H$. pylori infection, or any endoscopic finding. Our results demonstrate up-regulation of COX-2 in EGM, suggesting a possible malignant potential of this so-called harmless mucosa.
\end{abstract}

\section{Introduction}

Ectopic gastric mucosa (EGM) is an asymptomatic anomaly found in the cervical esophagus, also designated inlet patch. Macroscopically, the patch is characterized by reddish or

\section{Key words}

- Endoscopy

- Ectopic gastric mucosa

- Cyclooxygenase-2

- Barrett's esophagus

- Gastric biopsies salmon-red mucosa with a velvety appearance. It presents as either a single or multiple patches localized just below the upper esophageal sphincter. Microscopically, the patch can be lined with fundic or gastric body type glands (1-3). The prevalence of EGM ranges 
from 0.1 to $4.9 \%$ in most series, reaching $10 \%$ in a prospective study (1-3). The presence of Helicobacter pylori has been reported in 20 to $56.5 \%$ of cases (1-4).

The most accepted pathogenic theory considers EGM to be a congenital malformation. According to this theory, EGM represents the remnants of the columnar epithelium, secondary to a failure of the bidirectional replacement of the esophageal columnar epithelium by squamous mucosa, which starts in the mid-esophagus during the descending of the stomach in the fifth month of pregnancy (2).

There is no clear association between EGM and Barrett's esophagus, with contradictory published data. Some investigators believe that there is a possible link between these two entities $(5,6)$. On the other hand, some immunohistochemical studies have not confirmed this hypothesis (7).

EGM islets are generally assumed to be incidental findings, with no biopsies being routinely obtained and with patients not being referred to any surveillance program. Although rare, at least 23 cases of adenocarcinoma have been reported in the EGM (810).

The cyclooxygenase (COX) enzyme catalyzes the conversion of arachidonic acid to prostaglandins (11). Two COX isoforms have been identified in humans, COX-1 and COX2. COX-1 is a constitutively expressed enzyme, present in most tissues including the gastrointestinal tract, considered to be a housekeeping gene involved in general cell functions. COX-2 is an inducible enzyme usually undetectable in most tissues, upregulated in response to various stimuli, including growth factors, tumor promoters and cytokines (11-13).

Over-expression of COX-2 has been shown to have numerous effects on cells, such as increased cell proliferation, decreased apoptosis rate, stimulation of angiogenesis, and increased invasive and metastatic potential (13-18). Recent studies have demon- strated over-expression of COX-2 in Barrett's epithelium and associated adenocarcinoma $(14,19)$.

Furthermore, treatment with selective COX-2 inhibitors has been shown to reduce damage induced by acid and pepsin in the esophageal mucosa of rabbits and the incidence of tumors in an animal model of esophageal adenocarcinoma, to decrease proliferation and to induce apoptosis in both Barrett's epithelium and adenocarcinoma (14).

In the present study, we compared COX2 expression in biopsy specimens of heterotopic gastric mucosa, normal esophageal, gastric antrum and body mucosa, and Barrett's esophagus.

\section{Material and Methods}

The study was approved by our Institutional Human Research Review Committee. Consecutive patients referred for upper gastrointestinal endoscopy between August 2001 and May 2002 gave written informed consent and were enrolled in the study. Patients referred for emergency procedures were excluded.

\section{Endoscopic examination}

Upper endoscopy was performed after sedation with midazolam, meperidine or diazepam. All patients were prospectively assessed for the presence of EGM. Its macroscopic characteristics were documented as well as other endoscopic findings. Biopsies were obtained from the heterotopic mucosa. A biopsy from the gastric antrum was also taken in these patients to determine $H$. pylori infection by the rapid urease test (20).

Biopsy specimens obtained from the EGM were submitted to histological evaluation and to COX-2 determination by immunohistochemistry. Forty biopsy samples, including 10 from normal distal esophagus, 10 from normal gastric antrum, 10 from normal gastric body, and 10 from confirmed Barrett's 
esophagus (specialized intestinal epithelium and goblet cells) without dysplasia were also evaluated for the immunohistochemical expression of COX-2. These samples were retrieved from our tissue bank and matched to our EGM population for sex and age.

\section{Histopathological examination}

Biopsies were fixed in formaldehyde and embedded in paraffin and sections were stained with hematoxylin and eosin and modified Giemsa. Heterotopic gastric epithelium was classified as fundic, antral or mixed type. The presence of $H$. pylori was determined using the Giemsa stain.

\section{Immunohistochemistry}

Immunohistochemical studies were performed on formalin-fixed paraffin-embedded sections of all samples by the avidinbiotin peroxidase complex method.

Briefly, deparaffinized and rehydrated sections, pretreated by microwave antigen retrieval, were sequentially incubated with mouse anti-human COX-2 monoclonal antibody (Dako, Glostrup, Denmark) diluted 100 times with $1 \%$ swine serum in phosphatebuffered saline. The secondary antibody was biotinylated anti-mouse immunoglobulin (Dako). Finally, the sections were incubated with the avidin-biotin peroxidase complex (Dako). Color was developed using 3,3'diaminobenzide (Sigma, St. Louis, MO, USA - DS637) and sections were then counterstained with hematoxylin, dehydrated and mounted with permount. An esophageal adenocarcinoma specimen, previously shown to have strong COX-2 expression, was used as a positive control.

\section{Evaluation of immunohistochemical staining}

COX-2 immunohistochemical expression was reported according to a previously published score system (21). Staining intensity was graded as weak, 1, moderate, 2, or strong, 3. The stained area was classified as follows: $<10 \%$ of all cells stained in the cytoplasm as viewed by microscopy, $0 ; 10$ to $40 \%, 1 ; 40$ to $70 \%, 2 ;>70 \%, 3$. An average immunoreactive score was calculated by multiplying the staining intensity by the area of staining. A total score of 3 or more was defined as positive expression and a score of less than 3 as negative.

\section{Statistical analysis}

Data are reported as means $+\mathrm{SD}$ and as a box-plot graph. Comparison between groups was performed by the Student $t$-test and Fischer exact test for both continuous and categorical data (R 2.2.1 statistical software). The level of significance was set at $\mathrm{P}<0.05$.

\section{Results}

\section{Demographic characteristics of patients with} ectopic gastric mucosa

During the study period, 1327 patients were evaluated and EGM was encountered in $14(1.1 \%), 8$ of them men, with a median age of 47.4 years (range: 22 to 84 years). Only $2 / 14$ patients $(14.2 \%)$ with patches reported upper esophageal or laryngopharyngeal symptoms (burning in the throat and hoarseness).

\section{Endoscopic characteristics of ectopic gastric mucosa}

Most patients $(10 / 14,71.4 \%)$ presented only one EGM patch ranging in size from 2 to $20 \mathrm{~mm}$ (mean size: $6.5 \mathrm{~mm}$ ). The surface was smooth, regular and velvety in all cases (Figure 1).

Other endoscopic findings detected in these patients were: gastritis $(8 / 14)$, duodenal ulcer (4/14), peptic esophagitis (3/14), hiatal hernia (2/14), and portal hypertension (1/14). No Barrett's esophagus was found. 
Figure 1. Endoscopic appearance of ectopic gastric mucosa (arrow) with a smooth, regular and velvety islet of columnar tissue in the upper esophagus.

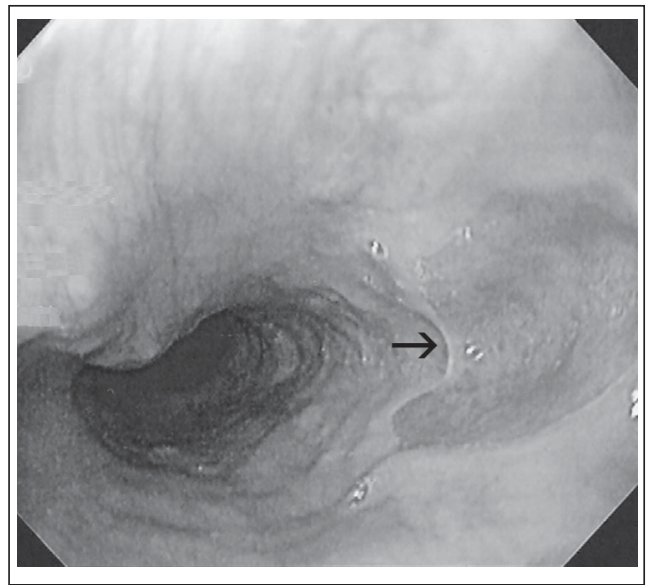

Table 1. Histological characteristics of ectopic gastric mucosa.

$\mathrm{N}(\%)$

\section{Epithelium type}

Fundic

$7(58.3 \%)$

Antral/fundic

$5(41.7 \%)$

Chronic inflammatory changes

Absent

$4(33.3 \%)$

Inactive

$3(25.0 \%)$

Mild

Helicobacter pylori (Giemsa)

Present

$5(41.7 \%)$

$6(50.0 \%)$

Other histological findings

Intestinal metaplasia

$2(16.6 \%)$

Table 2. Characteristics of ectopic gastric mucosa associated with Helicobacter pylori (HP) infection in 12 patients.

\begin{tabular}{lll}
\hline & HP positive & HP negative \\
\hline $\begin{array}{l}\text { Epithelium type } \\
\text { Fundic }\end{array}$ & 2 & 3 \\
$\quad$ Antral/fundic & 4 & 3 \\
Chronic inflammatory process & & \\
$\quad$ Absent & 1 & 3 \\
$\quad$ Inactive & 0 & 3 \\
$\quad$ Mild & 5 & $0^{*}$ \\
Intestinal metaplasia & & \\
$\quad$ Present & 1 & 1 \\
$\quad$ Absent & 5 & 5 \\
Helicobacter pylori (Giemsa) & & \\
$\quad$ Present & 6 & 3 \\
Absent & 0 & 3 \\
\hline *P $<0.01$ compared to HP-positive mucos (chi-square test).
\end{tabular}

${ }^{*} \mathrm{P}<0.01$ compared to HP-positive mucosa (chi-square test).
The ectopic patch group did not present more endoscopic findings suggestive of gastroesophageal reflux disease (hiatal hernia, esophagitis and Barrett's esophagus) than the patients without EGM patches $(\mathrm{P}=0.16)$.

Histological characteristics of ectopic gastric mucosa

Because of technical problems, histological and immunohistochemical examinations were performed in only 12 cases. The histological results are summarized in Table 1.

Hematoxylin and eosin staining revealed fundic type mucosa in $7 / 12$ cases $(58.3 \%)$ and mixed (antral/fundic) in 5/12 (41.7\%). Mild chronic inflammation was found in 5/ 12 patients (41.7\%), inactive chronic inflammation in $3 / 12(25 \%)$, and absence of inflammation in 4/12 (33.3\%).

H. pylori was identified in the ectopic epithelium in half the cases (6/12). All patients colonized with the bacteria in the inlet patch were also infected in the antrum, as confirmed by a positive rapid urease test.

Of 9 patients with confirmed antral $H$. pylori infection, 6 (66.7\%) also had $H$. pylo$r i$ in the patch. No correlation between $H$. pylori infection and the type of epithelium, the presence of intestinal metaplasia, or antral colonization could be established (Table 2). However, the presence of chronic inflammation was significantly correlated with H. pylori infection in the EGM $(\mathrm{P}=0.01)$, according to the chi-square test.

Two cases of intestinal metaplasia in the ectopic mucosa were identified and confirmed by the presence of specialized intestinal epithelium and goblet cells.

\section{Immunohistochemistry}

As shown in Figure 2A, COX-2 was undetectable in normal mucosa samples. Increased COX-2 expression was detected in $41.7 \%$ of EGM specimens (Figure 2B and C) and in 90\% (9/10) of Barrett's epithelium 
specimens (Figure 2D).

The comparison of COX-2 expression between normal mucosa and ectopic gastric mucosa reached statistical significance $(\mathrm{P}=$ $0.04)$ as also did the comparison between EGM and Barrett's esophagus $(\mathrm{P}=0.03)$. Average COX-2 expression scores (21) in EGM and Barrett's esophagus were 2.1 (2.3) and 6.1 (2.7), respectively (Figure 3).

We attempted to identify factors that could contribute to COX-2 expression in EGM. There was no difference related to age or gender. The presence of endoscopic findings of gastroesophageal reflux disease (GERD) was not associated with COX-2 immunoexpression, nor was epithelium type, presence of chronic inflammation, or $H$. pylori infection (Table 3 ).

\section{Discussion}

Heterotopic gastric mucosa is defined as a salmon pink colored patch localized in the upper esophagus and also designated as inlet patch. The prevalence of EGM in our study was $1.1 \%$, consistent with published reports (1-3). Symptoms occur in 6.2 to $20 \%$ of cases, mainly globus sensation, hoarseness, odynophagia, dysphagia, or oropharyngeal burning (2).

The macroscopic and microscopic characteristics of the inlet patches were similar to those previously reported. Diameter ranged from 2 to $50 \mathrm{~mm}$, although in most cases it was smaller than $10 \mathrm{~mm}(1-3,7)$. Presentation is usually as a single lesion (42 to $85 \%$ ), but occasionally two or more distinct patches can be observed (1-3,7). In our study, most cases presented a single patch $(71.4 \%)$ and average size was $6.5 \mathrm{~mm}$ (range 2 to $20 \mathrm{~mm}$ ), with $78.6 \%$ of lesions measuring $10 \mathrm{~mm}$ or less.

Fundic type mucosa was more frequent than antral fundic type mucosa $(58.3 \mathrm{vs}$ $41.7 \%$ ) and chronic inflammation was found in $66.7 \%$ of cases, consistent with previous reports $(1-3,7)$. Intestinal metaplasia was

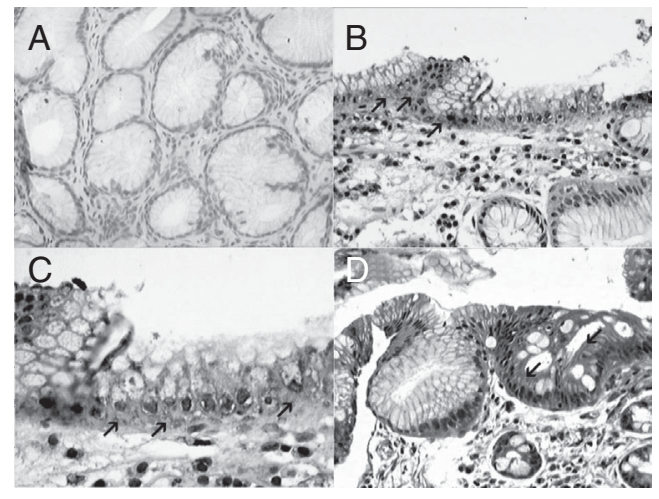

Figure 2. Examples of cyclooxygenase-2 (COX-2) immunoreactivity. $A$, No COX-2 expression in the normal specimen (200X). $B$, Immunohistochemical staining in ectopic gastric mucosa (arrows; 200X), and $C$, detail of the positive immunoexpression in the same specimen (400X), represented by a gray layer (arrows). $D$, COX-2 immunoexpression in Barrett's esophagus metaplastic epithelium (200X), demonstrated by a higher intensity gray layer (arrows).

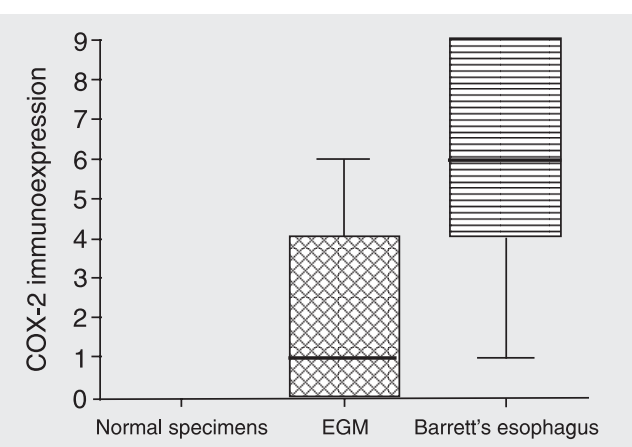

Figure 3. Box-plot showing the average distribution of cyclooxygenase-2 (COX-2) Yamauchi et al. (21) score in 30 normal specimens, 12 ectopic gastric mucosa (EGM) and 10 Barrett's esophagus. (Error bars are \pm SD and horizontal lines are medians). The value obtained for normal specimens was zero, since there was no COX-2 expression in the normal tissue.

Table 3. Comparison of various characteristics of ectopic gastric mucosa specimens regarding cyclooxygenase-2 (COX-2) immunoexpression.

\begin{tabular}{lcc}
\hline Characteristics & COX-2 positive & COX-2 negative \\
\hline $\begin{array}{l}\text { Gender } \\
\quad \text { Male:female }\end{array}$ & $4: 1$ & \\
Age (years) & & \\
$\quad$ Mean & 38 & 56 \\
$\quad$ Range & $18-67$ & $40-84$ \\
Associated endoscopic condition & & \\
$\quad$ Gastritis & $1(20 \%)$ & $6(85.7 \%)^{*}$ \\
$\quad$ Duodenal ulcer & 0 & $2(28.6 \%)$ \\
$\quad$ Esophagitis & $2(40 \%)$ & $1(14.3 \%)$ \\
$\quad$ Hiatal hernia & $1(20 \%)$ & $1(14.3 \%)$ \\
$\quad$ Portal hypertension & $1(20 \%)$ & 0 \\
Epithelium type & $2(40 \%)$ & $5(71.4 \%)$ \\
$\quad$ Fundic & $3(60 \%)$ & $2(28.6 \%)$ \\
$\quad$ Antral/fundic & & $3(42.9 \%)$ \\
Chronic inflammatory changes & $1(20 \%)$ & 0 \\
$\quad$ Absent & $3(60 \%)$ & $4(57.1 \%)$ \\
$\quad$ Inactive & $1(20 \%)$ & $5(71.4 \%)$ \\
$\quad$ Mild & $1(20 \%)$ & $2(28.6 \%)$ \\
Helicobacter pylori & 0 & \\
$\quad$ Present & & \\
Intestinal metaplasia & & \\
\hline
\end{tabular}

Data are reported as number with percent in parentheses unless otherwise indicated. $\mathrm{P}<0.05$ compared to COX-2-positive specimens (chi-square test). 
observed in $16.6 \%$ of patients, a slightly higher prevalence than previously reported (1-3).

EGM colonization by $H$. pylori has been described in 5.3 to $56.5 \%$ of patients $(1-4,7)$. In this study, it was present in half the cases and was closely related to the presence of chronic inflammation $(\mathrm{P}=0.01)$. We found no association with $H$. pylori antral infection. The presence of the bacteria was not correlated with mucosal type or with the presence of metaplasia.

Barrett's esophagus was not identified in any patient and GERD endoscopic findings did not correlate with the presence of an inlet patch.

EGM has been considered to be an incidental finding during upper endoscopy. However, some complications have been described, such as dysphagia due to rings or benign strictures $(22,23)$, ulceration (24), bleeding (25), esophago-tracheal fistulae (26), or perforation (27).

EGM is considered to be embryologic defect pathogenetically distinct from Barrett's esophagus. Contradictory data have been published about the immunohistochemical profiles of these two entities. Similarities have been demonstrated in the mucin profile (5), cytokeratin 7 and 20 pattern (28), and mucin core protein (28).

Adenocarcinomas developing on the EGM of the cervical esophagus are extremely rare and only few isolated cases have been reported (8-10). COX-2 is over-expressed in Barrett's esophagus and is involved in the sequence of events that lead to malignant transformation (19). The main objective of the present study was to determine COX-2 immunoexpression by the EGM and to compare it with the expression observed in normal esophagogastric and Barrett's esophagus samples.

The potential involvement of COX-2 in gastrointestinal carcinogenesis has been extensively discussed in the literature. Several studies have confirmed COX-2 over-expres- sion in colorectal cancer $(11,21,29)$ gastric adenocarcinoma $(13,16,30)$ and esophageal adenocarcinoma (19).

Experimental studies have demonstrated that COX-2 inhibits cell apoptosis, increases cell growth and promotes angiogenesis, contributing to tumorigenesis and increasing tumor invasiveness and metastatic potential (13-18). In vitro studies have suggested that COX-2 is able to suppress apoptosis and to exacerbate tumorigenic risk $(14,17)$. Cells over-expressing COX-2 contain increased amounts of anti-apoptotic protein bcl-2 (16). Selective in vitro COX-2 inhibition has been associated with significantly suppressed cell growth (proliferation) and increased apoptosis in esophageal adenocarcinoma cell lines $(14,31)$.

Increased invasiveness has been attributed to the modulation of cell adhesion by COX-2. The expression of adhesion proteins is decreased in cells over-expressing COX-2. These cell lines also have altered adhesion to structural proteins. Both factors seem to decrease cell adhesion and to increase invasive potential (17). Another possible important mechanism is the activation and formation of metalloproteinases 1 and 2 within cells expressing COX-2. These enzymes digest the collagen matrix of the basement membrane, thus stimulating the invasive and proliferative properties of tumor cells (18).

In vitro studies have confirmed the antiproliferative and pro-apoptotic effects promoted by selective inhibition of COX-2 in Barrett's adenocarcinoma and also in esophageal squamous carcinoma cell lines (14).

Buskens et al. (12) found negative or only weak COX-2 expression in the squamous epithelium of the esophagus. Lagorce et al. (19) identified over-expression in Barrett's esophagus compared to normal esophageal epithelium, unrelated to the degree of dysplasia and suggested that increased COX-2 expression plays an early role in the process of neoplastic transformation of 
Barrett's mucosa and may contribute to tumor formation. COX-2 expression is correlated with more aggressive clinicopathological tumor features, especially metastatic potential and poor prognosis (12).

We have detected high COX-2 expression in EGM. Immunoexpression of COX-2 was lower in EGM than in Barrett's esophagus specimens. Our results indicate a progressive over-expression of COX-2 from normal esophageal and gastric mucosa to EGM and Barrett's esophagus.

COX-2 immunoexpression was not related to gender, age, epithelium type, presence of chronic inflammation or intestinal metaplasia, $H$. pylori infection, or any of the associated endoscopic findings.

In the present study, COX-2 expression was not related to endoscopic GERD find- ings $(\mathrm{P}=0.22)$. We found no correlation between $H$. pylori infection and COX-2 expression in EGM. To our knowledge, there are no published data concerning COX-2 expression in heterotopic gastric mucosa.

Although the number of patients was small, our results confirmed high COX-2 expression in heterotopic mucosa, which could be interpreted as an eventual potential of neoplastic transformation of a so-called harmless mucosa.

Further studies are necessary to clarify the real malignant potential of this ectopic mucosa. Studies involving larger numbers of patients and a long follow-up will be necessary to confirm these results and to define the procedure to be followed. However, as of now, it may be advisable to perform a biopsy.

\section{References}

1. Akbayir N, Alkim C, Erdem L, Sokmen HM, Sungun A, Basak T, et al. Heterotopic gastric mucosa in the cervical esophagus (inlet patch): endoscopic prevalence, histological and clinical characteristics. J Gastroenterol Hepatol 2004; 19: 891-896.

2. Jacobs E, Dehou MF. Heterotopic gastric mucosa in the upper esophagus: a prospective study of 33 cases and review of literature. Endoscopy 1997; 29: 710-715.

3. Maconi G, Pace F, Vago L, Carsana L, Bargiggia S, Bianchi PG. Prevalence and clinical features of heterotopic gastric mucosa in the upper oesophagus (inlet patch). Eur J Gastroenterol Hepatol 2000; 12: 745-749.

4. Gutierrez O, Akamatsu T, Cardona H, Graham DY, El-Zimaity HM. Helicobacter pylori and heterotopic gastric mucosa in the upper esophagus (the inlet patch). Am J Gastroenterol 2003; 98: 12661270.

5. Bogomoletz WV, Geboes K, Feydy P, Nasca S, Ectors N, Rigaud C. Mucin histochemistry of heterotopic gastric mucosa of the upper esophagus in adults: possible pathogenic implications. Hum Pathol 1988; 19: 1301-1306.

6. Avidan B, Sonnenberg A, Chejfec G, Schnell TG, Sontag SJ. Is there a link between cervical inlet patch and Barrett's esophagus? Gastrointest Endosc 2001; 53: 717-721.

7. Feurle GE, Helmstaedter V, Buehring A, Bettendorf U, Eckardt VF. Distinct immunohistochemical findings in columnar epithelium of esophageal inlet patch and of Barrett's esophagus. Dig Dis Sci 1990; 35: 86-92.

8. Alrawi SJ, Winston J, Tan D, Gibbs J, Loree TR, Hicks W, et al. Primary adenocarcinoma of cervical esophagus. J Exp Clin Cancer Res 2005; 24: 325-330.
9. Abe T, Hosokawa M, Kusumi T, Kusano M, Hokari K, Kagaya $H$, et al. Adenocarcinoma arising from ectopic gastric mucosa in the cervical esophagus. Am J Clin Oncol 2004; 27: 644-645.

10. Hirayama N, Arima M, Miyazaki S, Shimada H, Okazumi S, Matsubara $\mathrm{H}$, et al. Endoscopic mucosal resection of adenocarcinoma arising in ectopic gastric mucosa in the cervical esophagus: case report. Gastrointest Endosc 2003; 57: 263-266.

11. Eberhart CE, Dubois RN. Eicosanoids and the gastrointestinal tract. Gastroenterology 1995; 109: 285-301.

12. Buskens CJ, Ristimaki A, Offerhaus GJ, Richel DJ, van Lanschot JJ. Role of cyclooxygenase-2 in the development and treatment of oesophageal adenocarcinoma. Scand J Gastroenterol Suppl 2003; 87-93.

13. Saukkonen K, Rintahaka J, Sivula A, Buskens CJ, Van Rees BP, Rio MC, et al. Cyclooxygenase-2 and gastric carcinogenesis. APMIS 2003; 111: 915-925.

14. Souza RF, Shewmake K, Beer DG, Cryer B, Spechler SJ. Selective inhibition of cyclooxygenase-2 suppresses growth and induces apoptosis in human esophageal adenocarcinoma cells. Cancer Res 2000; 60: 5767-5772.

15. von Rahden BH, Stein HJ, Puhringer F, Koch I, Langer R, Piontek G, et al. Coexpression of cyclooxygenases (COX-1, COX-2) and vascular endothelial growth factors (VEGF-A, VEGF-C) in esophageal adenocarcinoma. Cancer Res 2005; 65: 5038-5044.

16. Chen XL, Su BS, Sun RQ, Zhang J, Wang YL. Relationship between expression and distribution of cyclooxygenase-2 and bcl-2 in human gastric adenocarcinoma. World J Gastroenterol 2005; 11: 12281231.

17. Tsujii M, Dubois RN. Alterations in cellular adhesion and apoptosis 
in epithelial cells overexpressing prostaglandin endoperoxide synthase 2. Cell 1995; 83: 493-501.

18. Tsujii M, Kawano S, Dubois RN. Cyclooxygenase-2 expression in human colon cancer cells increases metastatic potential. Proc Natl Acad Sci U S A 1997; 94: 3336-3340.

19. Lagorce C, Paraf F, Vidaud D, Couvelard A, Wendum D, Martin A, et al. Cyclooxygenase-2 is expressed frequently and early in Barrett's oesophagus and associated adenocarcinoma. Histopathology 2003; 42: 457-465.

20. Thillainayagam AV, Arvind AS, Cook RS, Harrison IG, Tabaqchali S, Farthing MJ. Diagnostic efficiency of an ultrarapid endoscopy room test for Helicobacter pylori. Gut 1991; 32: 467-469.

21. Yamauchi $T$, Watanabe $M$, Kubota $T$, Hasegawa $H$, Ishii $Y$, Endo $T$, et al. Cyclooxygenase-2 expression as a new marker for patients with colorectal cancer. Dis Colon Rectum 2002; 45: 98-103.

22. McBride MA, Vanagunas AA, Breshnahan JP, Barch DB. Combined endoscopic thermal electrocoagulation with high dose omeprazole therapy in complicated heterotopic gastric mucosa of the esophagus. Am J Gastroenterol 1995; 90: 2029-2031.

23. Waring JP, Wo JM. Cervical esophageal web caused by an inlet patch of gastric mucosa. South Med J 1997; 90: 554-555.

24. Byrne M, Sheehan K, Kay E, Patchett S. Symptomatic ulceration of an acid-producing oesophageal inlet patch colonized by Helicobacter pylori. Endoscopy 2002; 34: 514.

25. Bataller R, Bordas JM, Ordi J, Llach J, Elizalde JI, Mondelo F. Upper gastrointestinal bleeding: a complication of "inlet patch mucosa" in the upper esophagus. Endoscopy 1995; 27: 282.

26. Kohler B, Kohler G, Riemann JF. Spontaneous esophagotracheal fistula resulting from ulcer in heterotopic gastric mucosa. Gastroenterology 1988; 95: 828-830.

27. Sanchez-Pernaute A, Hernando F, Diez-Valladares L, Gonzalez O, Perez AE, Furio $V$, et al. Heterotopic gastric mucosa in the upper esophagus ("inlet patch"): a rare cause of esophageal perforation. Am J Gastroenterol 1999; 94: 3047-3050.

28. Lauwers GY, Mino M, Ban S, Forcione D, Eatherton DE, Shimizu M, et al. Cytokeratins 7 and 20 and mucin core protein expression in esophageal cervical inlet patch. Am J Surg Pathol 2005; 29: 437 442.

29. Xiong B, Sun TJ, Hu WD, Cheng FL, Mao M, Zhou YF. Expression of cyclooxygenase-2 in colorectal cancer and its clinical significance. World J Gastroenterol 2005; 11: 1105-1108.

30. Yu LZ, Gao HJ, Bai JF, Sun G, Zhao HL, Sun L, et al. Expression of COX-2 proteins in gastric mucosal lesions. World $J$ Gastroenterol 2004; 10: 292-294.

31. Cheong E, Ivory K, Doleman J, Parker ML, Rhodes M, Johnson IT. Synthetic and naturally occurring COX-2 inhibitors suppress proliferation in a human oesophageal adenocarcinoma cell line (OE33) by inducing apoptosis and cell cycle arrest. Carcinogenesis 2004; 25: $1945-1952$ 